\title{
EXISTENCE AND MULTIPLICITY OF SOLUTIONS FOR A CLASS OF SUPERLINEAR $p$-LAPLACIAN EQUATIONS
}

\author{
JUAN WANG AND CHUN-LEI TANG
}

Received 16 May 2006; Revised 5 July 2006; Accepted 6 July 2006

By a variant version of mountain pass theorem, the existence and multiplicity of solutions are obtained for a class of superlinear $p$-Laplacian equations: $-\Delta_{p} u=f(x, u)$. In this paper, we suppose neither $f$ satisfies the superquadratic condition in AmbrosettiRabinowitz sense nor $f(x, t) /|t|^{p-1}$ is nondecreasing with respect to $|t|$.

Copyright (c) 2006 J. Wang and C.-L. Tang. This is an open access article distributed under the Creative Commons Attribution License, which permits unrestricted use, distribution, and reproduction in any medium, provided the original work is properly cited.

\section{Introduction and main results}

In this paper, we consider the following superlinear $p$-Laplacian equations with Dirichlet boundary value condition:

$$
-\triangle_{p} u=f(x, u), \quad x \in \Omega, u=0, x \in \partial \Omega,
$$

where $\triangle_{p} u$ is the $p$-Laplacian operator: $\triangle_{p} u=\operatorname{div}\left(|\nabla u|^{p-2} \nabla u\right)$ with $p>1, \Omega$ is a bounded domain in $R^{N}(N \geq 1)$ with smooth boundary $\partial \Omega, f \in C(\bar{\Omega} \times R, R)$ is subcritical in $t$, that is, there is a $q \in(p, n p /(n-p))$ when $N>p ; q \in(p,+\infty)$ when $N \leq p$ such that

$$
\lim _{t \rightarrow \infty} \frac{f(x, t)}{|t|^{q-1}}=0
$$

uniformly in a.e. $x \in \bar{\Omega}$. We are interested in the case that $f$ is superlinear in $t$ at infinity, that is,

$$
\lim _{|t| \rightarrow \infty} \frac{f(x, t) t}{|t|^{p}}=+\infty
$$

Ambrosetti and Rabinowitz have got the solutions of problem (1.1) by a very famous mountain pass theorem in [1]. But they supposed the well-known $(A R)$ condition holds, 
that is, for some $\mu>p, M>0$,

$$
0<\mu F(x, s) \leq f(x, s) s \quad \forall|s| \geq M, x \in \Omega \text {. }
$$

It is easy to see that the $(A R)$ implies (1.3). This $(A R)$ condition usually plays a very important role in verifying that the functional corresponding to problem has a MountainPass geometry and shows that a related $(P S)_{c}$ sequence is bounded (see $\left.[1,2,5,12]\right)$. But there are always many functions that do not satisfy $(A R)$ condition. Many efforts have been made to overcome the difficulties brought by the absence of the $(A R)$ (see $[3,6,8-$ $11,14,15,18-20])$. To the authors' knowledge, the following Assumption 1.1 is widely used (see $[8,18-20])$.

Assumption 1.1. $f(x, t) /|t|^{p-1}$ is nondecreasing with respect to $|t|$.

In this paper, we will get the existence of at least two nontrivial solutions of problem (1.1) where the nonlinearity $f(x, t)$ satisfies neither the classic $(A R)$ nor Assumption 1.1, instead, we suppose that an assumption weaker than Assumption 1.1 holds.

Assumption 1.2. There exists $\theta \geq 1$ such that $\theta G(x, t) \geq G(x, s t)$ for all $x \in \bar{\Omega}, t \in R$, and $s \in[0,1]$, where $G(x, t)=f(x, t) t-p F(x, t)$ and $F(x, t)=\int_{0}^{t} f(x, s) d s$.

Then our main results are the following two theorems.

Theorem 1.3. Suppose $f(x, t)$ is subcritical in $t$ and satisfies Assumption 1.2 and the following conditions hold:

(f1) $f(x, t) \geq 0$ for all $t \geq 0, x \in \bar{\Omega}$ and $f(x, t) \equiv 0$ for all $t \leq 0, x \in \bar{\Omega}$;

(f2) $\limsup \sup _{t \rightarrow 0^{+}}\left(f(x, t) / t^{p-1}\right)=a(x)$ and $\lim _{t \rightarrow+\infty}\left(f(x, t) / t^{p-1}\right)=+\infty$ uniformly in a.e. $x \in \bar{\Omega}$, where $a \in L^{\infty}(\bar{\Omega})$ satisfies $a(x) \leq \lambda_{1}$ for all $x \in \bar{\Omega}$ and $a(x)<\lambda_{1}$ on some $\Omega^{\prime} \subset \Omega$ with $\left|\Omega^{\prime}\right|>0, \lambda_{1}$ is the first eigenvalue of $-\Delta_{p}$ and $\left|\Omega^{\prime}\right|$ is the measure of $\Omega^{\prime}$.

Then problem (1.1) has at least one solution $u>0$.

If we consider a more general situation, we can get the following theorem.

Theorem 1.4. Suppose $f(x, t)$ is subcritical in $t$ and satisfies Assumption 1.2 and the following conditions hold:

(f3) $f(x, t) t \geq 0$ for all $t \in R, x \in \bar{\Omega}$;

(f4) $\limsup _{t \rightarrow 0}\left(f(x, t) t /|t|^{p}\right)=c(x)$ and $\lim _{|t| \rightarrow \infty}\left(f(x, t) t /|t|^{p}\right)=+\infty$ uniformly in a.e. $x \in \bar{\Omega}$, where $c \in L^{\infty}(\bar{\Omega})$ satisfies $c(x) \leq \lambda_{1}$ for all $x \in \bar{\Omega}$ and $c(x)<\lambda_{1}$ on some $\Omega_{0} \subset \Omega$ with $\left|\Omega_{0}\right|>0$.

Then problem (1.1) has at least two nontrivial solutions in which one is positive and the other is negative.

Remark 1.5. Assumption 1.2 was first introduced by Jeanjean in [6] for $p=2$, and recently by Liu and $\mathrm{Li}$ in [9] for general $p>1$. We can easily prove that Assumption 1.2 is equivalent to Assumption 1.1 when $\theta=1$ and Assumption 1.2 gives some general sense of monotony when $\theta>1$. Liu and Li in [9] has proved that Assumption 1.1 implies Assumption 1.2 when $p>1$. Moreover, we can find some examples that satisfy Assumption 1.2 but not Assumption 1.1. 
Example 1.6. Set $p=2$,

$$
f(x, t)=5 t \ln \left(1+t^{2}\right)+9 \sin t
$$

it follows that

$$
G(x, t)=9(t \sin t+2 \cos t-2)+5\left(t^{2}-\ln \left(1+t^{2}\right)\right) .
$$

Let $\theta=100$, we can prove by some simple computation that $G$ satisfies Assumption 1.2 but does not satisfy Assumption 1.1 any more.

Remark 1.7. We only consider the solutions of problem (1.1) in superlinear case. Recently, Zhou has got a positive solution of problem (1.1) for $p=2$ in [18] (see [18, Corollary 2.3]) and [19] (see [19, Theorem 1.2]). Then Li and Zhou extend the results to $p>1$ in [8] (see [8, Remark 1.2]). But in their discussion, they suppose (f1), Assumption 1.1, and the following condition hold:

(f5) $\lim _{t \rightarrow 0^{+}}\left(f(x, t) / t^{p-1}\right)=p(x)$ and $\lim _{t \rightarrow+\infty}\left(f(x, t) / t^{p-1}\right)=+\infty$ uniformly in a.e. $x \in$ $\Omega$, where $p(x) \equiv l \in\left[0, \lambda_{1}\right)$ (in $[8,18]$ ) or $p \in L^{\infty}(\Omega)$ with $\|p\|_{\infty}<\lambda_{1}$ (in [19]).

We can see that we extend the results of $[8,18,19]$ in superlinear case on two hands. On one hand, our condition (f2) is weaker than (f5), we do not need $\lim _{t \rightarrow 0^{+}}\left(f(x, t) / t^{p-1}\right)$ exist but only suppose $\lim \sup _{t \rightarrow 0^{+}}\left(f(x, t) / t^{p-1}\right)=a(x) \leq \lambda_{1}$ and $a(x)<\lambda_{1}$ on some $\Omega^{\prime} \subset$ $\Omega$ with positive measure, so we extend the range of the nonlinearity largely. On the other hand, from Remark 1.5 we can see that our Assumption 1.2 is weaker than Assumption 1.1 and we believe that Assumption 1.2 can take the place of Assumption 1.1 in many discussions of superlinear $p$-Laplacian problem. So our results are even new when $p=2$, we extend the results of $[8,18,19]$ in superlinear case for general $p>1$.

Remark 1.8. Liu and Li in [9] has got infinitely many solutions of problem (1.1) by the fountain theorem. But in their discussion, they supposed that $f(x, t)$ is odd with respect to $t$. In our discussion, we do not suppose $f(x, t)$ is odd any more. We will get the existence and multiplicity of solutions for problem (1.1) by a variant version of mountain pass theorem (introduced in [13] and used in [4], see also Lemma 2.1). So our results are different from those in [9].

Remark 1.9. Schechter and Zou have got a nontrivial solution of problem (1.1) for $p=2$ in [14] under the following superquadratic conditions $\left(a_{1}\right)$ together with $\left(a_{2}\right)$ or $\left(a_{2}^{\prime}\right)$ :

$\left(a_{1}\right)$ either

$$
\frac{F(x, t)}{t^{2}} \longrightarrow \infty \text { as } t \longrightarrow \infty
$$

or

$$
\frac{F(x, t)}{t^{2}} \longrightarrow \infty \quad \text { as } t \longrightarrow-\infty
$$

$\left(\mathrm{a}_{2}\right)$ there are constants $\mu_{1}>2, r>0$, and $C>0$ such that

$$
\mu_{1} F(x, t)-t f(x, t) \leq C\left(t^{2}+1\right), \quad|t| \geq r ;
$$

$\left(\mathrm{a}_{2}^{\prime}\right)$ the function $G(x, t)=f(x, t) t-2 F(x, t)$ is convex in $t$. 
It is easy to see that the function $f$ in Example 1.6 satisfies our Assumption 1.2 and $\left(a_{1}\right)$, but not satisfies $\left(a_{2}\right)$ nor $\left(a_{2}^{\prime}\right)$. In fact, if $G(x, t)>0$ for all $t \neq 0$, we can get Assumption 1.2 from $\left(a_{1}\right)$ and $\left(a_{2}^{\prime}\right)$. So our results are different from those of [14].

\section{Some important lemmas}

To look for a nontrivial solution of (1.1), we need the following version of the mountain pass theorem.

Lemma 2.1 (Schechter [13]). Let E be a real Banach space with its dual space $E^{*}$ and suppose that $J \in C^{1}(E, R)$ satisfies the condition

$$
\max \left\{J(0), J\left(u_{1}\right)\right\} \leq \alpha<\beta \leq \inf _{\|u\|=\rho} J(u)
$$

for some $\alpha<\beta, \rho>0$ and $u_{1} \in E$ with $\left\|u_{1}\right\|>\rho$. Let $c$ be characterized by

$$
c=\inf _{\gamma \in \Gamma} \max _{0 \leq \tau \leq 1} J(\gamma(\tau))
$$

where $\Gamma=\left\{\gamma \in C([0,1], E): \gamma(0)=0 ; \gamma(1)=u_{1}\right\}$ is the set of continuous paths joining 0 and $u_{1}$. Then there exists a sequence $\left\{u_{n}\right\} \subset E$ such that

$$
\begin{gathered}
J\left(u_{n}\right) \longrightarrow c \geq \beta \quad(n \longrightarrow+\infty), \\
\left(1+\left\|u_{n}\right\|\right)\left\|J^{\prime}\left(u_{n}\right)\right\|_{E^{*}} \longrightarrow 0 \quad(n \longrightarrow+\infty) .
\end{gathered}
$$

In the proof of the theorems we will use the following lemma to prove the geometric condition of the mountain pass theorem.

LEMma 2.2. If (f2) holds, there exits a positive constant $\alpha<1$ such that

$$
\int_{\Omega} a(x)|u|^{p} d x<\alpha \int_{\Omega}|\nabla u|^{p} d x
$$

for all $u \in W_{0}^{1, p}(\Omega)$.

Proof. Let us prove it by contradiction. Otherwise, there exists a sequence $\left\{u_{n}\right\} \subset W_{0}^{1, p}(\Omega)$ such that

$$
\int_{\Omega} a(x)\left|u_{n}\right|^{p} d x \geq\left(1-\frac{1}{n}\right) \int_{\Omega}\left|\nabla u_{n}\right|^{p} d x
$$

Set $v_{n}=u_{n} /\left\|u_{n}\right\|$, it follows that

$$
\int_{\Omega} a(x)\left|v_{n}\right|^{p} d x \geq 1-\frac{1}{n}
$$

Then by (f2) and the Poincare inequality, we have

$$
\int_{\Omega} a(x)\left|v_{n}\right|^{p} d x \leq \lambda_{1} \int_{\Omega}\left|v_{n}\right|^{p} d x \leq \int_{\Omega}\left|\nabla v_{n}\right|^{p} d x=\left\|v_{n}\right\|^{p}=1 .
$$


Therefore, we obtain

$$
1-\frac{1}{n} \leq \int_{\Omega} a(x)\left|v_{n}\right|^{p} d x \leq \lambda_{1} \int_{\Omega}\left|v_{n}\right|^{p} d x \leq \int_{\Omega}\left|\nabla v_{n}\right|^{p} d x=1 .
$$

For $\left\{v_{n}\right\}$ is bounded in $W_{0}^{1, p}(\Omega)$, then there exists $v \in W_{0}^{1, p}(\Omega)$ such that

$$
\begin{gathered}
v_{n} \longrightarrow v \quad \text { weakly in } W_{0}^{1, p}(\Omega), \\
v_{n} \longrightarrow v \quad \text { in } L^{p}(\Omega) .
\end{gathered}
$$

Let $n \rightarrow \infty$ in (2.8), one gets

$$
\begin{gathered}
\lambda_{1} \int_{\Omega}|v|^{p} d x=\lim _{n \rightarrow \infty} \int_{\Omega}\left|\nabla v_{n}\right|^{p} d x=1, \\
\int_{\Omega}\left(a(x)-\lambda_{1}\right)|v|^{p} d x=0 .
\end{gathered}
$$

By (2.10), the weakly lower semicontinuity of $\|\cdot\|_{p}$ and the Poincare inequality we have

$$
1=\liminf _{n \rightarrow \infty} \int_{\Omega}\left|\nabla v_{n}\right|^{p} d x \geq \int_{\Omega}|\nabla v|^{p} d x \geq \lambda_{1} \int_{\Omega}|v|^{p} d x=1
$$

it follows that

$$
\lambda_{1} \int_{\Omega}|v|^{p} d x=\int_{\Omega}|\nabla v|^{p} d x=1
$$

From (2.13), we can see that $v$ is in fact the eigenfunction corresponding to the first eigenvalue of the following problem:

$$
-\triangle_{p} u=\lambda|u|^{p-2} u
$$

Then from the results for $p$-Laplacian, we have $v \neq 0$, so (2.11) implies that $a(x)=\lambda_{1}$ a.e. on $\Omega$, but this is impossible by (f2). Hence, Lemma 2.2 holds.

To see that a nonnegative solution of problem (1.1) is in fact a positive solution in $\Omega$, we need the following strong maximum principle for $p$-Laplacian.

Lemma 2.3 (Vázquez [17]). Let $u \in C^{1}(\Omega)$ be such that $\triangle_{p} u \in L_{\text {loc }}^{2}(\Omega), u \geq 0$ a.e. on $\Omega$, $\triangle_{p} u \leq \beta(u)$ a.e. with $\beta:[0, \infty] \rightarrow R$ continuous nondecreasing, $\beta(0)=0$ and either $\beta(s)=0$ for some $s>0$ or $\beta(s)>0$ for all $s>0$, but

$$
\int_{0}^{1} j(s)^{-1 / p} d s=\infty, \quad \text { where } j(s)=\int_{0}^{s} \beta(t) d t
$$

holds. Then if $u$ does not vanish identically of $\Omega$, it is positive everywhere in $\Omega$. 


\section{Proof of the theorems}

Proof of Theorem 1.3. It is well known that to seek a nontrivial weak solution of problem (1.1) is equivalent to finding a nonzero critical point of the $C^{1}$-function:

$$
J(u)=\frac{1}{p} \int_{\Omega}|\nabla u|^{p} d x-\int_{\Omega} F(x, u) d x .
$$

In the following proof, we will find the critical points of $J(u)$ in three steps.

Step 1. There exist some $\rho, \beta>0$, such that $J(u) \geq \beta$ for all $u \in W_{0}^{1, p}(\Omega)$ with $\|u\|=\rho$.

In fact, by Lemma 2.2 , let $\varepsilon>0$ be small enough such that $\alpha+\varepsilon / \lambda_{1}<1$. Since $f(x, t)$ is subcritical and (f2) holds, there exist $\delta_{1}, \delta_{2}>0$ and $M>0$ for the above $\varepsilon$, such that

$$
\begin{gathered}
F(x, s) \leq \frac{1}{p}(a(x)+\varepsilon)|s|^{p} \quad \forall|s|<\delta_{1}, x \in \bar{\Omega}, \\
F(x, s) \leq \frac{1}{p} \varepsilon|s|^{q} \quad \forall|s|>\delta_{2}, x \in \bar{\Omega}, \\
F(x, s) \leq M|s|^{p} \leq \frac{M}{\delta_{1}^{q-p}}|s|^{q} \quad \forall \delta_{1} \leq|s| \leq \delta_{2}, x \in \bar{\Omega},
\end{gathered}
$$

where $q$ is the same as in (1.2). Set $A=\max \left\{(1 / p) \varepsilon, M / \delta_{1}^{q-p}\right\}>0$, then we have

$$
F(x, s) \leq \frac{1}{p}(a(x)+\varepsilon)|s|^{p}+A|s|^{q}
$$

for all $(x, s) \in \bar{\Omega} \times R$. By the Poincare inequality and Sobolev inequality, one obtains

$$
\begin{aligned}
J(u) & \geq \frac{1}{p}\|u\|^{p}-\frac{1}{p} \int_{\Omega}(a(x)+\varepsilon)|u|^{p} d x-A \int_{\Omega}|u|^{q} d x \\
& \geq \frac{1}{p}\|u\|^{p}-\frac{1}{p} \int_{\Omega}\left(\alpha+\frac{\varepsilon}{\lambda_{1}}\right)|\nabla u|^{p} d x-C\|u\|^{q} \\
& =\frac{1}{p}\left(1-\alpha-\frac{\varepsilon}{\lambda_{1}}\right)\|u\|^{p}-C\|u\|^{q},
\end{aligned}
$$

where $C>0$ is a constant. Since $1-\alpha-\varepsilon / \lambda_{1}>0$ and $p<q$, let $\rho$ be small enough such that

$$
\beta \triangleq \frac{1}{p}\left(1-\alpha-\frac{\varepsilon}{\lambda_{1}}\right) \rho^{p}-C \rho^{q}>0
$$

so we have $\left.J\right|_{\partial B_{\rho}} \geq \beta>0$.

Step 2. There exists $e \in W_{0}^{1, p}(\Omega)$ with $\|e\|>\rho$ such that $J(e)<0$.

Since $\lim _{t \rightarrow+\infty}\left(f(x, t) / t^{p-1}\right)=+\infty$ by (f2), then for any $\varepsilon>0$, there exists $M>0$ such that $f(x, t) / t^{p-1} \geq 1 / \varepsilon$ for all $t>M$ and $x \in \bar{\Omega}$. Set $c(\varepsilon)=(1 / \varepsilon) M^{p-1}$, consequently,

$$
f(x, t) \geq \frac{1}{\varepsilon} t^{p-1}-c(\varepsilon)
$$


for all $t \geq 0$ and $x \in \bar{\Omega}$, which implies that

$$
f(x, s t) t \geq \frac{1}{\varepsilon} s^{p-1} t^{p}-c(\varepsilon) t
$$

for all $x \in \bar{\Omega}, t \geq 0$, and $0 \leq s \leq 1$. Integrating both sides of the inequality (3.7) on $[0,1]$ with respect to $s$, we obtain

$$
F(x, t) \geq \frac{1}{p \varepsilon} t^{p}-c(\varepsilon) t
$$

for all $t \geq 0$. It follows from (3.8) that

$$
F\left(x, t \varphi_{1}\right) \geq \frac{1}{p \varepsilon} t^{p} \varphi_{1}^{p}-c(\varepsilon) t \varphi_{1}
$$

Dividing by $t^{p}$, one has

$$
\frac{F\left(x, t \varphi_{1}\right)}{t^{p}} \geq \frac{1}{p \varepsilon} \varphi_{1}^{p}-\frac{c(\varepsilon) \varphi_{1}}{t^{p-1}}
$$

thus we have

$$
\int_{\Omega} \frac{F\left(x, t \varphi_{1}\right)}{t^{p}} d x \geq \int_{\Omega}\left(\frac{1}{p \varepsilon} \varphi_{1}^{p}-\frac{c(\varepsilon) \varphi_{1}}{t^{p-1}}\right) d x .
$$

Let $t \rightarrow \infty$ in (3.11), it follows that

$$
\liminf _{t \rightarrow+\infty} \int_{\Omega} \frac{F\left(x, t \varphi_{1}\right)}{t^{p}} d x \geq \int_{\Omega} \frac{1}{p \varepsilon} \varphi_{1}^{p} d x
$$

for all $\varepsilon>0$. For $\varepsilon>0$ is arbitrary, let $\varepsilon \rightarrow 0$, then one obtains

$$
\lim _{t \rightarrow+\infty} \int_{\Omega} \frac{F\left(x, t \varphi_{1}\right)}{t^{p}} d x=+\infty
$$

Consequently,

$$
\frac{J\left(t \varphi_{1}\right)}{t^{p}}=\frac{1}{p}\left\|\varphi_{1}\right\|^{p}-\int_{\Omega} \frac{F\left(x, t \varphi_{1}\right)}{t^{p}} d x \longrightarrow-\infty \quad(t \longrightarrow+\infty) .
$$

Hence, let $t_{0}$ be big enough and $e=t_{0} \varphi_{1}$, then we have $J(e)<0$.

Define

$$
\Gamma=\left\{\gamma \in C\left([0,1], W_{0}^{1, p}(\Omega)\right): \gamma(0)=0 ; \gamma(1)=e\right\}, \quad c=\inf _{\gamma \in \Gamma 0 \leq \tau \leq 1} \max _{0}(\gamma(\tau)),
$$

then $c \geq \beta>0$. By Lemma 2.1, there exists a sequence $\left\{u_{n}\right\} \subset W_{0}^{1, p}(\Omega)$, such that

$$
\begin{gathered}
J\left(u_{n}\right)=\frac{1}{p} \int_{\Omega}\left|\nabla u_{n}\right|^{p} d x-\int_{\Omega} F\left(x, u_{n}\right) d x \rightarrow c \quad(n \rightarrow \infty), \\
\left(1+\left\|u_{n}\right\|\right)\left\|J^{\prime}\left(u_{n}\right)\right\| \longrightarrow 0 \quad(n \longrightarrow \infty) .
\end{gathered}
$$


8 A class of superlinear $p$-Laplacian equations

Combining (3.16) and (3.17), we obtain

$$
\int_{\Omega}\left(\frac{1}{p} f\left(x, u_{n}\right) u_{n}-F\left(x, u_{n}\right)\right) d x=c+o(1) .
$$

Step 3. Let us prove that the sequence $\left\{u_{n}\right\}$ is bounded.

Otherwise, there is a subsequence of $\left\{u_{n}\right\}$ (still denoted by $\left\{u_{n}\right\}$ ) satisfying $\left\|u_{n}\right\| \rightarrow \infty$ as $n \rightarrow \infty$. Set $w_{n}=u_{n} /\left\|u_{n}\right\|$, then $w_{n}$ is bounded. So we may assume that for some $w \in$ $W_{0}^{1, p}(\Omega)$ there is a subsequence of $\left\{w_{n}\right\}$ (still denoted by $\left.\left\{w_{n}\right\}\right)$ such that

$$
\begin{gathered}
w_{n} \longrightarrow w \quad \text { weakly in } W_{0}^{1, p}(\Omega), \\
w_{n} \longrightarrow w \quad \text { in } L^{r}(\Omega)\left(1 \leq r<p^{*}\right), \\
w_{n}(x) \longrightarrow w(x) \quad \text { a.e. } x \in \Omega,
\end{gathered}
$$

as $n \rightarrow \infty$. It is easy to see that $w_{n}^{+}$and $w_{n}^{-}$have the same convergence which is similar to (3.19) where $u^{+}=\max \{u, 0\}$ and $u^{-}=\min \{u, 0\}$ for $u \in W_{0}^{1, p}(\Omega)$.

On one hand, we claim that $w^{+} \equiv 0$. Otherwise, set $\Omega_{1}=\left\{x \in \Omega: w^{+}(x)=0\right\}, \Omega_{2}=$ $\left\{x \in \Omega: w^{+}(x)>0\right\}$. Since $\left\|u_{n}\right\| \rightarrow+\infty$, we have $u_{n}^{+} \rightarrow+\infty$ as $n \rightarrow \infty$ for a.e. $x \in \Omega_{2}$. Since

$$
\lim _{t \rightarrow+\infty} \frac{f(x, t)}{|t|^{p-1}}=+\infty
$$

we have

$$
\lim _{n \rightarrow+\infty} \frac{f\left(x, u_{n}^{+}\right)}{\left(u_{n}^{+}\right)^{p-1}}=+\infty \quad \text { a.e. on } \Omega_{2} .
$$

If $\left|\Omega_{2}\right|>0$, one obtains

$$
\begin{aligned}
o(1) & =\left\langle J^{\prime}\left(u_{n}\right), u_{n}\right\rangle=\int_{\Omega}\left|\nabla u_{n}\right|^{p} d x-\int_{\Omega} f\left(x, u_{n}\right) u_{n} d x \\
& \leq\left\|u_{n}\right\|^{p}-\int_{\Omega_{2}} f\left(x, u_{n}^{+}\right) u_{n}^{+} d x=\left\|u_{n}\right\|^{p}\left(1-\int_{\Omega_{2}} \frac{f\left(x, u_{n}^{+}\right)}{\left(u_{n}^{+}\right)^{p-1}}\left(w_{n}^{+}\right)^{p}\right) d x
\end{aligned}
$$

So it follows that

$$
o(1) \leq 1-\int_{\Omega_{2}} \frac{f\left(x, u_{n}^{+}\right) u_{n}^{+}}{\left(u_{n}^{+}\right)^{p-1}}\left(w_{n}^{+}\right)^{p} d x
$$

By Fatou's lemma, we have

$$
1 \geq \liminf _{n \rightarrow \infty} \int_{\Omega_{2}} \frac{f\left(x, u_{n}^{+}\right)}{\left(u_{n}^{+}\right)^{p-1}}\left(w_{n}^{+}\right)^{p} d x=+\infty,
$$

which is a contradiction, so $\left|\Omega_{2}\right|=0$ and $w^{+} \equiv 0$. 
On the other hand, if $w^{+} \equiv 0$, set a sequence $\left\{t_{n}\right\}$ of real numbers such that $J\left(t_{n} u_{n}\right)=$ $\max _{t \in[0,1]} J\left(t u_{n}\right)$. For any integer $m>0$, set $w_{n}^{m}=(2 p m)^{1 / p} w_{n}$. By (f2), (3.3), and the convergence of $w_{n}^{+}$, one has

$$
\begin{aligned}
\limsup _{n \rightarrow \infty} \int_{\Omega} F\left(x, w_{n}^{m}\right) d x & =\limsup _{n \rightarrow \infty} \int_{\Omega} F\left(x,(2 p m)^{1 / p} w_{n}^{+}\right) d x \\
& \leq \limsup _{n \rightarrow \infty}\left(\int_{\Omega} 2 m\left(\lambda_{1}+\varepsilon\right)\left(w_{n}^{+}\right)^{p} d x+\int_{\Omega} A(2 p m)^{q / p}\left(w_{n}^{+}\right)^{q} d x\right) \\
& =\limsup _{n \rightarrow \infty}\left(C_{1}\left\|w_{n}^{+}\right\|_{p}^{p}+C_{2}\left\|w_{n}^{+}\right\|_{q}^{q}\right) \\
& =C_{1}\left\|w^{+}\right\|_{p}^{p}+C_{2}\left\|w^{+}\right\|_{q}^{q}=0,
\end{aligned}
$$

where $C_{1}, C_{2}>0$ are constants. Since $\left\|u_{n}\right\| \rightarrow+\infty$ as $n \rightarrow \infty$, one has $0 \leq(2 p m)^{1 / p} /\left\|u_{n}\right\| \leq$ 1 when $n$ is big enough. By the definition of $t_{n}$, we obtain

$$
J\left(t_{n} u_{n}\right) \geq J\left(w_{n}^{m}\right) \geq 2 m-\int_{\Omega} F\left(x, w_{n}^{m}\right) d x \geq m,
$$

which implies that

$$
J\left(t_{n} u_{n}\right) \rightarrow+\infty \quad(n \longrightarrow \infty)
$$

Noting that $J(0)=0$ and $J\left(u_{n}\right) \rightarrow c$, so $0<t_{n}<1$ when $n$ is big enough. It follows that

$$
\int_{\Omega}\left|\nabla\left(t_{n} u_{n}\right)\right|^{p} d x-\int_{\Omega} f\left(x, t_{n} u_{n}\right) t_{n} u_{n} d x=\left\langle J^{\prime}\left(t_{n} u_{n}\right), t_{n} u_{n}\right\rangle=\left.t_{n} \frac{d J\left(t u_{n}\right)}{d t}\right|_{t=t_{n}}=0 .
$$

But for $0 \leq t_{n} \leq 1$, we have $\theta G\left(x, u_{n}\right) \geq G\left(x, t_{n} u_{n}\right)$ by Assumption 1.2, then (3.27) and (3.28) imply that

$$
\begin{aligned}
\int_{\Omega}\left(\frac{1}{p} f\left(x, u_{n}\right) u_{n}-F\left(x, u_{n}\right)\right) d x & =\frac{1}{p} \int_{\Omega} G\left(x, u_{n}\right) d x \geq \frac{1}{p \theta} \int_{\Omega} G\left(x, t_{n} u_{n}\right) d x \\
& =\frac{1}{\theta} \int_{\Omega}\left(\frac{1}{p} f\left(x, t_{n} u_{n}\right) t_{n} u_{n}-F\left(x, t_{n} u_{n}\right)\right) d x \\
& =\frac{1}{\theta} \int_{\Omega}\left(\frac{1}{p}\left|\nabla t_{n} u_{n}\right|^{p}-F\left(x, t_{n} u_{n}\right)\right) d x \\
& =\frac{1}{\theta} J\left(t_{n} u_{n}\right) \longrightarrow+\infty \quad(n \longrightarrow \infty) .
\end{aligned}
$$

which contradicts (3.18), so $\left\{u_{n}\right\}$ is bounded. 
By the compactness of Sobolev embedding and the standard procedures, we know that $\left\{u_{n}\right\}$ has a subsequence which converges to a weak solution $u \in W_{0}^{1, p}(\Omega)$ of (1.1). By (f1), we must have $u \geq 0$. By the regularity results of Ladyzhenskaya and Ural'tseva (see [7]), $u \in L^{\infty}$ and hence $u \in C_{\text {loc }}^{1, \alpha}(\Omega) \subset C^{1}(\Omega)$ (see [16]). Since $u \in L^{\infty}(\Omega)$, it is easy to see that $\triangle_{p} u=-f(x, u) \in L_{\text {loc }}^{2}(\Omega)$. Moreover, we have $-f(x, u) \leq 0$ by $(\mathrm{f} 1)$. Hence by Lemma 2.3 with $\beta(u)=0$, one has $u>0$ a.e. on $\Omega$. Then Theorem 1.3 is proved.

Proof of Theorem 1.4. First, let us consider the following truncated problem:

$$
-\triangle_{p} u=f_{1}(x, u), \quad x \in \Omega, u=0, x \in \partial \Omega,
$$

where

$$
f_{1}(x, t)=\left\{\begin{array}{cc}
f(x, t), & t \geq 0 \\
0, & t \leq 0
\end{array}\right.
$$

For this problem (3.30), it is easy to see that $f_{1}(x, t)$ satisfies the conditions of Theorem 1.3. So by Theorem 1.3, there is a positive solution $u>0$ of problem (3.30) and it is also a solution of problem (1.1) by the definition of $f_{1}$.

Next, let us see another truncated problem:

$$
-\triangle_{p} u=f_{2}(x, u), \quad x \in \Omega, u=0, x \in \partial \Omega,
$$

where

$$
f_{2}(x, t)= \begin{cases}f(x, t), & t \leq 0 \\ 0, & t \geq 0\end{cases}
$$

In order to find a solution of problem (3.32), set $v=-u, g(x, t)=-f_{2}(x,-t)$, then problem (3.32) is equivalent to the following problem:

$$
-\triangle_{p} v=g(x, v), \quad x \in \Omega, v=0, x \in \partial \Omega
$$

It is easy to see that if $v$ is a solution of problem (3.34), then $u=-v$ is a solution of problem (3.32). Since $f(x, t)$ satisfies the conditions in Theorem 1.4, $g(x, t)$ satisfies all the conditions in Theorem 1.3. Then by Theorem 1.3, there is a positive solution $v>0$ of problem (3.34), so $u=-v<0$ is a solution to problem (3.32) and it is also a solution of problem (1.1).

From the above discussion, we can deduce that there exist at least a positive solution and a negative solution of problem (1.1). Then problem (1.1) has at least two nontrivial solutions. 


\section{Acknowledgments}

The authors would like to thank the referee for valuable suggestions. This work was supported by National Natural Science Foundation of China (No. 10471113) and by the Teaching and Research Award Program for Outstanding Young Teachers in Higher Education Institutions of MOE, China.

\section{References}

[1] A. Ambrosetti and P. H. Rabinowitz, Dual variational methods in critical point theory and applications, Journal of Functional Analysis 14 (1973), no. 4, 349-381.

[2] H. Brézis and L. Nirenberg, Positive solutions of nonlinear elliptic equations involving critical Sobolev exponents, Communications on Pure and Applied Mathematics 36 (1983), no. 4, 437477.

[3] D. G. Costa and C. A. Magalhães, Variational elliptic problems which are nonquadratic at infinity, Nonlinear Analysis 23 (1994), no. 11, 1401-1412.

[4] D. G. Costa and O. H. Miyagaki, Nontrivial solutions for perturbations of the p-Laplacian on unbounded domains, Journal of Mathematical Analysis and Applications 193 (1995), no. 3, $737-$ 755.

[5] J. V. Goncalves and S. Meira, On a class of semilinear elliptic problems near critical growth, International Journal of Mathematics and Mathematical Sciences 21 (1998), no. 2, 321-330.

[6] L. Jeanjean, On the existence of bounded Palais-Smale sequences and application to a LandesmanLazer-type problem set on $\mathbf{R}^{N}$, Proceedings of the Royal Society of Edinburgh. Section A. Mathematics 129 (1999), no. 4, 787-809.

[7] O. A. Ladyzhenskaya and N. N. Ural'tseva, Linear and Quasilinear Elliptic Equations, Academic Press, New York, 1968.

[8] G. Li and H.-S. Zhou, Asymptotically linear Dirichlet problem for the p-Laplacian, Nonlinear Analysis, Series A: Theory Methods 43 (2001), no. 8, 1043-1055.

[9] S. B. Liu and S. J. Li, Infinitely many solutions for a superlinear elliptic equation, Acta Mathematica Sinica 46 (2003), no. 4, 625-630 (Chinese).

[10] K. Perera and M. Schechter, Semilinear elliptic equations having asymptotic limits at zero and infinity, Abstract and Applied Analysis 4 (1999), no. 4, 231-242.

[11] A. Qian, Existence of infinitely many nodal solutions for a superlinear Neumann boundary value problem, Boundary Value Problems (2005), no. 3, 329-335.

[12] P. H. Rabinowitz, Minimax methods and their application to partial differential equations, Seminar on Nonlinear Partial Differential Equations (Berkeley, Calif, 1983), Math. Sci. Res. Inst. Publ., vol. 2, Springer, New York, 1984, pp. 307-320.

[13] M. Schechter, A variation of the mountain pass lemma and applications, Journal of the London Mathematical Society. Second Series 44 (1991), no. 3, 491-502.

[14] M. Schechter and W. Zou, Superlinear problems, Pacific Journal of Mathematics 214 (2004), no. $1,145-160$.

[15] A. Szulkin and W. Zou, Homoclinic orbits for asymptotically linear Hamiltonian systems, Journal of Functional Analysis 187 (2001), no. 1, 25-41.

[16] P. Tolksdorf, Regularity for a more general class of quasilinear elliptic equations, Journal of Differential Equations 51 (1984), no. 1, 126-150.

[17] J. L. Vázquez, A strong maximum principle for some quasilinear elliptic equations, Applied Mathematics and Optimization 12 (1984), no. 3, 191-202.

[18] H.-S. Zhou, Existence of asymptotically linear Dirichlet problem, Nonlinear Analysis. Series A: Theory and Methods 44 (2001), no. 7, 909-918. 
12 A class of superlinear $p$-Laplacian equations

[19] An application of a mountain pass theorem, Acta Mathematica Sinica 18 (2002), no. 1, 27-36.

[20] W. Zou, Variant fountain theorems and their applications, Manuscripta Mathematica 104 (2001), no. 3, 343-358.

Juan Wang: Department of Mathematics, School of Mathematics and Statistics,

Southwest University, Chongqing 400715, China

E-mail address: ykuai@swu.edu.cn

Chun-Lei Tang: Department of Mathematics, School of Mathematics and Statistics,

Southwest University, Chongqing 400715, China

E-mail address: tangcl@swu.edu.cn 